Utah State University

DigitalCommons@USU

5-1977

\title{
Genetic Control of Spermiogenesis in Drosophila melanogaster: The Effects of Abnormal Association of Centrosome and Nucleus in Mutant MS(1)6S
}

Douglas Lee Habliston

Utah State University

Follow this and additional works at: https://digitalcommons.usu.edu/etd

Part of the Biology Commons

\section{Recommended Citation}

Habliston, Douglas Lee, "Genetic Control of Spermiogenesis in Drosophila melanogaster: The Effects of Abnormal Association of Centrosome and Nucleus in Mutant MS(1)6S" (1977). All Graduate Theses and Dissertations. 8312.

https://digitalcommons.usu.edu/etd/8312

This Thesis is brought to you for free and open access by the Graduate Studies at DigitalCommons@USU. It has been accepted for inclusion in All Graduate Theses and Dissertations by an authorized administrator of DigitalCommons@USU. For more information, please contact digitalcommons@usu.edu.

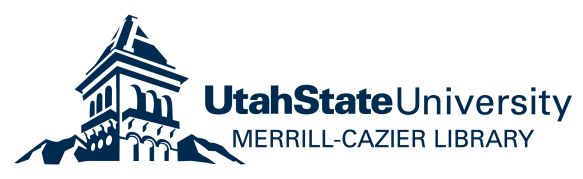


GENETIC CONTROL OF SPERMIOGENESIS

IN DROSOPHILA MELANOGASTER:

THE EFFECTS OF ABNORMAL ASSOCIATION

OF CENTROSOME AND NUCLEUS IN MUTANT MS(1)6S

by

Douglas Lee Habliston

A thesis submitted in partial fulfillment of the requirements for the degree

of

MASTER OF SCIENCE

in

Biology

Approved:

Major Professor

Committee Member

Committee Me $\overline{m b e r}$

Dean of Graduate School

UTAH STATE UNIVERSITY

Logan, Utah 


\section{ACKNOWLEDGEMENTS}

A very special thanks is extended to Dr. Hugh $P$. Stanley and Dr. James T. Bowman for cooperation, understanding and assistance despite unusual circumstances of time and distance.

I express appreciation to the National Science Foundation under contract Grant GS-22606 with Utah State University, for financial support.

I am most appreciative to my wife, Polly, for her help and cheerful encouragement throughout my thesis program.

Douglas L. Habliston 
TABLE OF CONTENTS

Page

TITLE ........................... . . ACKNOLWEDGEMENTS ...................

TABLE OF CONTENTS .................. . . iii LIST OF FIGURES ... . . . . . . . . . . . . . . iv ABSTRACT .......................

INTRODUCTION................................. vi MATERIALS AND METHODS ..................... 1

RESULTS ..................... . . 3

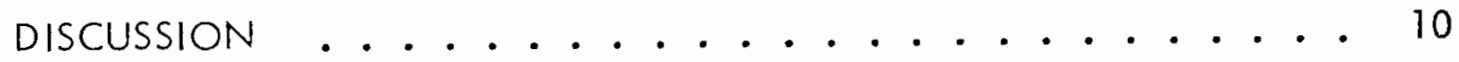

REFERENCES .............................. 16 


\section{LIST OF FIGURES}

Figure

1. Cross Section through bundle of mutant spermatids showing developmental characteristics of early Stage $6 . \ldots . .$.

2. Longitudinal section through the basal tip of a mutant

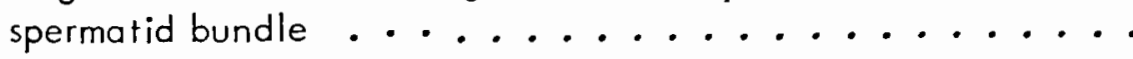

3. A portion of an early Stage 6 nucleus. .......... 21

4. Longitudinal section through the central portion of a Stage 6

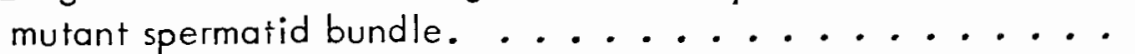

5. Section through several axonemes of late Stage 6 , all in a common cytoplasmic mass. ............... 22

6. Section through an aggregation of early spermatid mitchondria. 23

7. A section through a spermatid bundle. ......... 23

8. A longitudinal section through a mutant spermatid. ..... 24

9. A section through a mutant testis following treatment with

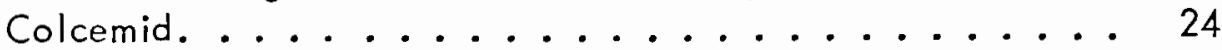




\begin{abstract}
Genetic Control of Spermiogenesis in Drosophila Melanogaster:
\end{abstract}

The Effects of Abnormal Association

of Centrosome and Nucleus in Mutant MS(1)6S

by

Douglas Lee Habliston, Master of Science

Utah State University, 1977

Major Professor: Dr. Hugh P. Stanley

Department: Biology

Mutant ms(1) 65 of Drosophila me lanogaster is characterized by a spectrum of abnormalities of spermatid differentiation. Included in these are failure of the basal body to make normal attachment to the nucleus, failure of acrosomes to develop, and lack of a normal pupulation of perinuclear microtubules. There is also a failure of normal chromatin condensation and nuclear elongation. The mitochondria do not form a normal nebenkern, but nevertheless fuse, elongate, and develop paracrystalline bodies. The number of normal axonemes is markedly reduced as a result of the failure of some centrioles to form basal bodies. All of these aberrations can be related to defective interactions of the "centrosome" or "microtubule-organizing-centre" with other organelles. The analysis of spermiogenesis in this mutant further clarifies the roles of microtubules in nuclear condensation and elongation and in cellular elongation in general. 


\section{INTRODUCTION}

In a series of studies on the differentiation of spermatids in malesterile mutants of Drosophila melanogaster $(8,12-14,23-26,39,40,42$,$) ,$ some insight has been gained into the functional roles of various organelles in spermiogenesis. Other investigators, studying the effects of chromosomal deletions or translocations $(10,11,36)$, have added information that is useful in unraveling the interactions that occur during this particularly complex cellular differentiation. The use of microtubule inhibitors such as Colcemid or vinblastine $(30,40)$ has also proved to be a fruitful approach to defining the parameters of cellular development.

Many of the mutants examined in the studies cited above have been characterized by a disruption of the proper orientation of cellular organelles, but in the present study, for the first time, multiple defects can be related to that focal point of cell organization, the centrosome. This mutant provides further information on the importance of microtubules in the condensation and shaping of the nucleus, in the elongation and shaping of the mitochondrial derivatives, and in general elongation of the spermatid cell. 


\section{MATERIALS AND METHODS}

The ethyl methanesulfonate-induced mutant used in this study, ms (1)6S, is a sex-linked male-sterile mutation isolated in our laboratory by $\mathrm{B}$. S. Shadbolt. Since its discovery, the mutant has been maintained in stock balanced with chromosome FM4(15). Although considered a sterile fly, ms(1)6S males very rarely produce a few functional sperm. The occasional development of viable spermatozoa is documented by the infrequent appearance of whiteeyed females, which is possible only as a result of successful crosses of balanced bar-eyed females of the genotype $\underline{w}$ ms (1)65/FM4 to $\underline{w}$ ms $(1) 65 / Y$ sterile males. All flies were maintained at $25 \pm 1 \mathrm{C}$ on standard corn mealsucrose-yeast-agar medium with propionic acid added as a mold inhibitor. In some experiments, newly-eclosed mutant imagos were placed in half pint bottles with a "Kinwipe" saturated with $0.25 \mathrm{mM}$ Colcemid (CIBA) in $1 \%$ sucrose for 48 hours (39).

Testes from treated and from newly-eclosed untreated mutant imagos were dissected in Karnovsky's fixative (9). This and all other solutions were buffered at $\mathrm{pH} 7.4$ with $0.2 \mathrm{M}$ cacodylate buffer. Following dissection, the testes were transferred to fresh fixative and allowed to stand at room temperature for an additional 2 hours. The testes were then rinsed twice for 20 minutes with buffer. After post-fixation in $2 \%$ osmium tetroxide in buffer for an additional 2 hours and two 20 -minute rinses in buffer, the testes were dehydrated in a graded acetone series. The tissues were then embedded in D.E.R $732 / 332$ epoxy resin according to the procedures of Lockwood (16). 
Isolated bundles were obtained by placing testes in $50 \mathrm{ml}$ of $0.2 \%$ buffered trypsin (Sigma) in a Belco digestion flask equipped with a small stirring bar. The testes were stirred very slowly for 20 minutes at $37 \mathrm{C}$. The digestion was effectively terminated by the addition of sterile calf serum. The mixture was decanted into $12 \mathrm{ml}$ conical centrifuge tubes and centrifuged at $1200 \mathrm{~g}$ for two minutes. The supernatant was decanted and tissue fixation was carried out in the centrifuge tubes as previously outlined. After infiltration with resin, the bundles were transferred with a Pasteur pipette to a $47 \mathrm{~mm}$ disc of D.E.R. 732/332 epoxy resin for embedding.

Sections were cut with diamond knives (Dupont) using a Sorvall PorterBl um II-B microtome, stained with uranyl acetate (38) and lead citrate (21), and examined with either a Zeiss EM-9S-2 or Jeol JEM 100-B electron microscope. For light microscopy, one-micron sections were stained using the procedure of Richardson et al. (22). Whole mounts were examined with a Zeiss Ultrophot light microscope.

Since the nucleus differentiates abnormally and axonemes are rare or missing in many sections, stage assignments are based entirely on the differentiation of mitochondrial derivatives. When axonemes are present, their stage of differentiation correlates with that of the mitochondrial derivatives.

All references to normal sperm development and stages of differentiation, unless otherwise noted, are to those reported by Stanley et al. (30). Regions of the testis follow the definitions of Tokuyasu (32). 
RESULTS

Mutant testes are of normal size and shape, with morphologically normal accessory glands and ducts. However, no motile sperm were found in squash preparations of the mutant testes in Drosophila Ringer's. Under phase optics, mutant testes were seen to contain a number of elongating bundles extending from area 2 of the apical portion to area 7 and 8 in the basal region. Bundles in areas 9 and 10 are tightly coiled and packed, as in the normal testis (33).

Bundles obtained by trypsin digestion of testes range from $0.25 \mathrm{~mm}$ to $2.2 \mathrm{~mm}$ in length. Many of the mutant bundles have localized swellings in the middle to mid-apical regions, in contrast to wild-type bundles which are of fairly constant diameter. Staining mutant bundles of approximately $1.5 \mathrm{~mm}$ length with fast green revealed that all nuclei in a bundle are located in these central swellings and that they are at a developmental stage comparable to early stage 6. In wild-type, the nuclei are all located at one end of the bundle.

One-micron cross-sections of embedded testes were observed to contain smaller numbers of bundle profiles than those reported for wild-type flies by Tokuyasu (32). Whereas averages of approximately 55 profiles in area 2 , 75 in area 5, and 65 in area 8 are typical of wild-type, about one-half these numbers were found in corresponding areas of mutant testes.

Electron microscopic observations revealed several abnormalities in the early differentiation of spermatids. In wild-type Drosophila melanogaster the functional transition from centriole to basal body involves a definitive association with the nuclear envelope in the early spermatid. Later, this association is modified so that the proximal portion of the 
basal body lies in a nuclear indentation closely surrounded by nuclear envelope, the centriolar adjunct material forms a ring around the basal body just outside the nuclear indentation, and axoneme formation is initiated at the distal end. During this time two other notable events occur: the mitochondria fuse to form the nebenkern, which divides into two approximately equal bodies along a plane determined by the location of the elongating axoneme, and the acrosomal vesicle comes to lie adjacent to the nucleus. Our observations of ms(1)6S spermatids have failed to show even one acrosomal vesicle profile or a single association of a basal body with a nucleus, and nebenkern formation does not proceed normally beyond the initial aggregation phase (31).

Cross-sections through the nuclear region of developing mutant spermatids are marked by the presence of nuclei, microtubules, axonemes, and mitochondrial derivatives (Fig. 1), but in no case were centrioles observed in contact with a nucleus. To locate the missing centrioles, bundles were sectioned at short intervals, and those centrioles which had formed basal bodies were all located at the same extreme end of the elongating bundle (Fig. 2). Careful sectioning failed to locate nuclei in the vicinity of the basal bodies. It was also observed that axonemes, when they do develop, generally terminate in the medial regions of the bundle. Therefore, in mutant spermatid bundles, the basal bodies define a polarity to which the nucleus does not conform.

A significant observation from the serial cross-sections is the reduced number of normal axonemes and the large number of disrupted axonemes present (Figs. 1,5). The largest number of normal axoneme profiles counted in any section of a single bundle was fifteen, and the largest number of identifiable disrupted axonemes was twenty-five. Thus, the number of axonemes present is 
significantly less than the expected wild-type figure of sixty-four.

No intact axonemes were found in the caudal quarter of the bundles although mitochondrial derivatives and cytoplasmic microtubules were present in this area. Rarely, however, centrioles devoid of centriolar adjuncts and axonemes were found in other regions of the cells. These, obviously, had not participated in the elongation of the bundle.

A numerically normal population of randomily distributed microtubules is present in the cytoplasm surrounding the Stage 6 nucleus (Fig. 3 ). In contrast to wild type, however, these microtubules are never concentrated in a single, large, nuclear concavity but, rather, occur around the entire surface of the mutant nucleus except for certain areas where the two membranes of the nuclear envelope are adherent. Cross-linking of adjacent microtubules, both around the nucleus and around mitochondrial derivatives lying close to the nucleus, does occur. However, neither the perinuclear microtubules nor the periderivative microtubules are normally aligned in a single row surrounding the individual organelles. Instead, they appear in groups that lie along the same axis as the elongating bundle. The perinuclear microtubules, even though occasionally establishing cross-links with other perinuclear microtubules, never establish normal contact with the nuclear envelope through ridges of dense material (30). In rare instances, nuclei were observed that had a closely associated microtubular sheath resembling that in wild-type spermatids. These nuclei most likely represent the rare viable sperm that were mentioned earlier.

Differentiation of mutant nuclei appears to progress normally through the first two or three stages of spermiogenesis. The chromatin condensation pattern, nuclear envelope characteristics, perinuclear endoplasmic reticulum association, and changes in shape proceed as expected in the absence of 
associated centrioles, microtubules, and acrosomes. Irregular nuclear patterns and shapes first begin to appear at Stage 5 of differentiation.

The coarse chromatin network that forms a wide band near the concave surface of the Stage 6 wild-type nucleus is reduced to a smaller band of thick fibers distributed around the periphery of an irregularly shaped mutant nucleus (Fig. 3). Condensation of chromatin material is never seen to proceed beyond this stage, although the development of paracrystalline material in the mitochondrial derivatives indicates more advanced stages of development in the latter organelles (Fig. 7). Figure 4 depicts in longitudinal section the most elongate nucleus observed in this mutant.

Nuclear pores, which are normally confined to one surface in wild-types Stage 5 and 6 nuclei, are distributed unevenly around the entire nuclear surface. At those random locations where the two leaflets of the nuclear envelope come into close apposition, nuclear pores are lacking (Fig. 3 ). Microtubules and condensed chromatin are also excluded from these areas of close membrane association, which is consistant with observations of normal Stage 6 nuclei. However, the close nuclear envelope leaflet apposition is random and of varying duration in the mutant, whereas in the normal Drosophila Stage 6 nucleus, this thickened nuclear envelope only occurs on the convex surface.

Although acrosomes were never observed, Golgi apparati are abundantly scattered throughout the cells, often associated with axonemes and nuclei or isolated in irregular cytoplasmic partitions (Fig. 5).

In ms(1)65 males, the aggregation of mitochondria is arrested prior to the formation of a nebenkern typical of Stage 2 (Fig. 6). No clew or onion stage (31) is ever produced. However, there is considerable mitochondrial 
fusion forming profiles which in cross-section appear as rod-like segments with some branching. Fused segments were an average of 1.2 m by 6.1 um in size, forming mitochondrial aggregates approximately 17 um in diameter. The next definable stage of mitochondrial differentiation approximates Stage 5 except that the mitochondrial aggregate has not divided to form primary and secondary derivatives. Rather the aggregate encloses areas of cytoplasm containing polyribosomes and other granular material. The rodlike segments have fused to form one or more large derivatives of varying size that have begun to elongate in association with a population of cytoplasmic microtubules. By early Stage 6 , these multiple derivatives are in contact with the networks of smooth endoplasmic reticulum (Fig. 1). The points of contact with the endoplasmic reticulum are marked by formation of small paracrystalline bodies (Fig. 5).

The association of derivatives with axonemes, when present, is usually abnormal. The derivatives often do not assume the relationship of one primary and one secondary derivative to each axoneme. Rather, multiple derivatives, virtually all of which form paracrystalline bodies, may contact the sheath of a single axoneme (Fig. 5). When two or more axonemes occur in close proximity, one of several alternative configurations is observed. There may be the normal attachment of a derivative to one periaxonemal sheath, attachment of one derivative to two or more adjacent periaxonemal sheaths, or, finally, no derivative-periaxonemal sheath union at any point (Figs. 1,5$)$.

Elongation of derivatives, however, can progress in the absence of close axonemal association. That this elongation follows the course of cytoplasmic microtubules is clear from longitudinal sections (Fig. 8). Cross-sections show that groups of microtubules occur in the vicinity of most derivatives (Figs. 3, 5, 7). That elongation is accompanied by continuing fusion of 
mitochondria is suggested by the reduction of the number of derivative profiles in the later stages. Instead of the normal 64 primary and 64 secondary derivatives present in late wild type bundles, an average of only 45 identifiable primary and 10 putative secondary derivatives are seen. However, none of these observations are inconsistent with the hypothesis that all derivatives in this mutant are capable of forming paracrystalline bodies. By chance some profiles of nebenkern derivatives wilf lack paracrystalline bodies because the latter are discontinuous in their early formation stages.

As may be expected in a mutant such as ms (1)6S in which populations of microtubules are either lacking or abnormally located and grouped, the process of cytoplasmic separation (individualization (33)) in the spermatids is faulty, resulting in isolated axonemes unaccompanied by other organelles, separate cytoplasmic masses containing only groups of cytoplasmic microtubules, masses containing derivatives and microtubules, and still others with no formed structures other than ribosomes (Fig. 1).

A simple experiment modeled after that of Wilkinson (39) was conducted to determine the respective roles of the microtubules, axonemes, and nebenkern derivatives in the process of elongation. Mutant flies were treated with colcemid and their testes were compared with those of untreated mutant controls. The most significant effect of colcemid treatment (39) is the blocking of elongation by disrupting cytoplasmic microtubules and axonemes. As expected, when introduced to mutant ms (1)6S flies, the spindle poison prevents formation of all axonemes and cytoplasmic microtubules not present before treatment began. Some older bundles, which have completed most of their differentiation prior to treatment are located in the basal portion of the testis and show partial elongation. Instead of nomal elongate 
bundles, ovoid bodies containing abnormal sperm were found throughout the apical portion of the testis. In these, though the derivatives do not elongate, they do fuse to some extent, often producing circular or spiral derivatives (Fig. 9). 


\section{DISCUSSION}

Van Beneden (37), Boveri (2), and Wilson (41) regarded the centrosome as a distinct and persistent cell organelle. Physiologically, it was regarded as the special organ of cell division and the dynamic center of the cell. Van Beneden noted that structural polarity may appear either in the external form of the cell or as a polarized grouping of the various cell components about an ideal axis determined by the position of the centrosome. Boveri regarded the centrosome as the special fertilizing element in the spermatozoon which, when introduced into the egg, endowed the latter with the power of division and development. More recently, Pickett-Heaps (20) has postulated the existence of a "microtubule-organizingcentre" as the focal point of division spindle assembly. Wilson emphasized the role that the centriole plays in motile sperm of plants and animals and its involvement in many cell activities that have no immediate connection with cell division.

The juxtanuclear position of the centrosome in spermatids has long been used as an indication of polarity and symmetry of the cell (6). More recently, Fawcett et al. (7) have drawn attention to an imaginary line passing through the nucleus and centrosome which defines the cell axis. Our data show that even if, as in ms(1)6S, the centriole is not in juxtaposition to the nucleus, it sometimes persists in establishing polarity and the axis of elongation. The other cellular organelles do not necessarily conform to this polarity except as dictated by mechanical constraints effected by the elongation of the sperm bundle. This conclusion is consistent with evidence for centrosomal organization of spermatid polarity in two vertebrate species studied by Stanley $(28,29)$. 
Three observations are pertinent to an interpretation of the very early events which lead to the observed basal-body abnormalities. First, the breeding data show that normal associations between the centriole and nucleus must occur rarely, although they have not been observed in thin sections. Second, the functional transition from centriole to basal body is an event of reduced probability in ms(1)6S. Third, centrioles are either transformed into basal bodies and participate in axoneme elaboration and bundle elongation or they remain entirely centriolar in structure, located randomly in the cel1. Consistent with but not required by these observations, is the postulate that in ms(1)6S transient associations between nuclei and centrioles occur which, if effective, induce the transition to a basal body, but which in $\underline{\text { ms (1) } 65}$ are only rarely stabilized in the wild-type structural configuration as described by Tokuyasu (35).

The chromatoid body has been postulated by several authors to store RNA for protein synthesis $(17,18,4)$. An ontogenic correlation between the chromatoid body (centriolar adjunct (3)) and acrosomal complex formation has been established by Soderstrom and Parvinen (27). They claim the chromatoid body in rat spermatids establishes a transient contact with intranuclear material during early spermiogenesis, as well as with the Golgi complex. In this way, the chromatid body participates in the formation of the acrosomal system by transferring protein or RNA information from the nucleus to the Golgi apparatus, which in turn produces the acrosome material. A similar set of-interactions in normal Drosophila and their failure in the mutant would explain the lack of acrosomal vesicles or mature acrosomes in ms (1)6S. clearly, mutant basal bodies organize microtubules and form elongating axonemes. Wilkinson $(39,40)$ has documented the importance of these structures in Drosophila spermiogenesis including their role in cell elongation. 
However, it has been difficult to delineate the respective roles of the axoneme, the caudal cytoplasmic microtubules, and the primary nebenkern derivative in the achievement and maintenance of spermatid elongation. Certain properties of these organelles in $\underline{\mathrm{ms}}(1) 6 \mathrm{~S}$ clarify their respective roles. That the nebenkern derivative alone cannot mediate cell elongation is shown by the failure of wild-type bundles to elongate in the presence of Colcemid of vinblastine $(39,40)$ and the similar results obtained with Colcemid-treated ms(1)6S. However, in untreated, elongating mutant bundles, cytoplasmic microtubules and nebenkern derivatives are found through the entire length of the cells whereas axonemes are missing from the caudal one-quarter. This observation is most easily explained if the cytoplasmic microtubules are the primary mediators of elongation and direct or constrain the path of the developing axoneme and nebenkern derivative, which, when mature, constitute a stable, semi-rigid system. This interpretation is entirely consistent with Wilkinson's (39) and Tokuyasu's (34) observations as well as with the lack of peripheral microtubules in normal mature sperm (30)

The nuclear abnormalities of ms(1)6S are strikingly similar to those observed by Shoup $(25,26)$ and Wilkinson et al. $(39,40)$ in Drosophila spermatids developing with abnormal populations of perinuclear microtubules. Although it is obvious that other factors affect nuclear morphogenesis in some species (5), the case for the critical role of microtubules in Drosophila is convincing. In ms(1)6S, the persistence of large, medially located, irregularly shaped nuclei with chromatin no more condensed than that characteristic of wild-type Stage 6 strongly supports this contention.

That the cytoplasmic microtubules organized by the centriole are of two different functional types has been reported by wilkinson et al. (40). 
One group extends cephalad around the nucleus from the centriolar adjunct whereas the other group extends caudad in close proximity to the two nebenkern derivatives. In ms(1)6s it appears that the functional group normally recognized as perinuclear microtubules is reduced or absent due to the position of the basal body and centriolar adjunct at the extreme cephalic end of the cell, removed from any contact with the nucleus. Thus, no perinuclear microtubules make contact with the nuclear envelope through dense ridges to influence the condensation of chromatin or to act in shaping the nucleus. Since the posteriorly developing microtubules from the centriole are seen in serial cross section along the entire length of a bundle, we believe that these are the same microtubules seen in juxtaposition with the nucleus in sections through the nuclear region. It is reasonable then to assume that this population of posteriorly lengthening cytoplasmic microtubules exerts some mechanical constriction which might account for the partial elongation and deformities of the nucleus noticed in the mutant. However, it is apparent that these caudal cytoplasmic microtubules cannot effectively replace the normal perinuclear microtubules in influencing later differentiation of the nucleus.

It is significant that though the centriole-nucleus relationship is almost never properly established in ms(1)6S, all the isogenous sperm cells in a bundle establish identical anterior-posterior polarity. There appears to be an intrinsic factor regulating the positioning of the centriole to establish proper orientation of the spermatocytes or early spermatids. The nucleus appears to play a passive role in the formation of polarity in the differentiating cell. This is interesting evidence indeed for the dynamic role of the centrosome. 
In wild-type spermiogenesis the spermatid mitochondria aggregate near the base of the developing flagellum and the subsequent division plane of the nebenkern is aligned with the flagellum. The two nebenkern derivatives subsequently elongate adjacent to doublets number 2 and 4 . The primary derivative is always that one adjacent to doublet number 2 . In similar fashion, most other insects exhibit nebenkern formation and subsequent division into two parts, which in some species may differentiate equally (19), or, in others, unequally as in ‥ melanogaster $(30,31)$. Since the primary and secondary derivatives consistently develop adjacent to the same two doublets of the axoneme, however, the possibility exists that some ininfluence relative to the axoneme determines which of two equipotential nebenkern derivatives becomes the primary and which the secondary body. The properties of mutants ms (2) TOR and ms(2)3R studied by Romrell $(23,24)$ tend to discount such an influence by specific portions of the axoneme. Both of these mutants show development of both primary and secondary derivatives that frequently bear no close physical relationship to axonemal doublets, the important factor for paracrystalline body formation being contact with ER membranes rather than contact with the axoneme per se $(2,23,24,39,40)$. Recently Bacetti (1) has postulated that paracrystalline body precursors are pumped into the primary derivative from the cisternum of the periaxonemal membranous sheath. This postulate implies that a different sort of membrane connection may exist in the primary as opposed to the secondary derivative. In mutant ms(1)6S the mitochondria undergo initial aggregation but do not proceed through the "clew" and "onion" stages (31) to produce two equalsized derivatives. Instead, the mitochondria fuse in apparent random manner, in some cases aligning themselves along the axes of microtubules before fusing. In the most advanced bundles observed, the number of derivative 
profiles was approximately the same as that found in wild type bundles. The late derivatives appear rather normal except for the fact that most if not all form paracrystalline bodies. They are then, by definition, primary derivatives. This implies that it is during the process of normal fusion and division that a distinction is made as to which of two derivatives will differentiate as the primary derivative and which will have this capability suppressed. In bypassing these crucial nebenkern stages, most or all of the mitochondria in ms(1)6S spermatids differentiate in the same way, namely as primary derivatives. It is a notable circumstance that even though the mitochondria skip these remarkably complex nebenkern stages, the final products so nearly approximate the normal condition. 


\section{REFERENCES}

1. BACCETTI, B. and AfZELIUS, B., The Biology fo the Sperm Cell. Monographs Developt. Biol. 10, 103(1976) Karger, Basel.

2. BOVERI, T., Jena. Zeitschr. XXI (1887).

3. BRELAND, 0.P., GASSNER, G., RIESS, R.W. and BIESELE, J.J., Con. J. Genet. Cytol. 8,759 (1966).

4. DAVIS, J.R., FIRLIT, C.F., Amer. J. Physiol. 209, 425 (1965).

5. DIRKSON, E.R. and STAPRONS, I., J. Cell Biol. 57, (1973).

6. FAWCETT. D.W., An Atlas of Fine Structure--The Cell Its Organelles and Inclusions, 49, W. B. Saunders Company, 1969.

7. FAWCETT, D.W., ANDERSON, W.A., and PHILLIPS, D.M., Develop. Biol. 26, 220 (1971).

8. HESS, O. and MEYER, G.F., Advan.Genet. 14, 171 (1968).

9. KARNOVSKY, M.J., J. Cell. Biol. 27, 137A (1965).

10. KESSEL, R. G., J. U1trastruct. Res. 16, 293 (1966).

11. KIEFER, B.I., Genetics 54, 1441 (1966).

12. __ ibid. 60, 192 (1968).

13. _ ibid. 61, 157 (1969).

14. J_ J. Cel1 Sci. 6, 177 (1970).

15. LINDSLEY; D.L. and GRELL, E.H., Carnegie Inst. Wash. Pub7. No. 627, (1968).

16. LOCKWOOD, W.R., Anat. Rec. 150, 129 (1964).

17. MONESI, V., J. Cell Biol. 22, 521 (1964).

18. ___ Exp. Ce11 Res. 39, 197 (1965).

19. PHILLIPS, D.M., J. Ce11 Biol. 44, 243 (1970).

20. PICKETT--HEAPS, J., Cytobios 3, 205 (1971).

21. REYNOLDS, E., J. Cell Biol. 17, 208 (1963).

22. RICHARDSON, K.C., JARETT, L. and FINKE, E.H., Stain Technol. 35, 313 (1960).

23. ROMRELL, L. J., STANLEY, H.P. and BOWMAN, J.T., J. U1trastruct. Res. 38, 563 (1972). 
24. ibid. 38,578 (1972).

25. SHOUP, J.R., J. Cell Biol. 31, 107A (1966).

26. ibid. 32, 663 (1967).

27. SODERSTROM, K., and PARVINEN, M., Cell and Tissue Res. 168, 335 (1976).

29. ibid. 36, 86 (1971).

30. STANLEY, H.P., BOWMAN, J.T., ROMRELL, L.J., REED, S.C. and WILKINSON, R.F., J. U1trastruct. Res. 41, 433 (1972).

31. TATES, A.D., Cytodifferentiation during spermatogenesis in Drosophila melanogaster. Thesis. Drukkerij J. H. Pasmans, 'S-Gravenhage (1971).

32. TOKUYASU, K.T., PEACOCK, W.J. and HARDY, R.W., Z. Zellforsch. 124, 479 (1972).

33. _- ibid. 127, 492 (1972).

34. Exp. Cell Res. 84, 239 (1974).

35. J. U1tra Res. 50, 117 (1975).

36. TURNER, R.F., J. Cell Biol. 46, 220 (1920).

37. VAN BENEDEN, E., Arch. de Biol. IV (1883).

38. WATSON, M. L., J. Biophys. Biochem. Cytol. 4, 475 (1958).

39. WILKINSON, R.F., STANLEY, H.P., and BOWMAN, J.T., J. Ultrastruct. Res. 48, $242(1974)$.

40. ibid. 53,354 (1975).

41. WILSON, E.B., The Cell In Development and Heredity, p. 30, 108. MacMillan and Company, New York, 1928.

42. LAUGHRAN, L.J., STANLEY, H.P., AND BOWMAN, J.T., J. Ultrastruct. Res. 56, 21 (1976). 
Figure 1. Cross section through a bundle of mutant spermatids showing developmental characteristics of early Stage 6 . Portions of three nuclei (N) are shown. Masses of cytoplasm containing membranes, many nebenkern derivatives (ND) and a few axonemes (A) are characteristic. Small masses of cytoplasm containing an axoneme, a nebenkern derivative, or no major organelles at all are seen among the larger masses (arrows 1). At the lower right, four axonemes are seen, two of which show three nebenkern derivatives attached to their periaxonemal sheaths (arrows 2). The nebenkern derivatives are just begining to develop paracrystalline bodies; one derivative (arrow 3 ) shows two such structures. $\times 18,900$.

Figure 2. Longitudinal section through the basal tip of a mutant spermatid bundle. The basal body (BB) with surrounding centriolar adjunct (CA) is positioned at the extreme tip of the cytoplasmic mass unconnected to a nucleus. $\times 64,700$.

Figure 3. A portion of an early Stage 6 nucleus illustrating the general distribution of the microtubules (MT), the condensation of chromatin (CH) adjacent to several areas of nuclear envelope containing nuclear pores, and the interspersed areas of adherent nuclear membranes with closely juxtaposed cytoplasmic membranes (unlettered arrows). $\times 48,000$.

Figure 4. Longitudinal section through the central portion of a Stage 6 mutant spermatid bundle. The nucleus shown $(N)$ is the most elongate, most differentiated and most nearly normal nucleus seen in this mutant. Above the nucleus are several profiles of nebenkern derivatives (ND) of late Stage 6 differentiation. x 13,000. 
Figure 5. Section through several axonemes of late Stage 6 , all in a common cytoplasmic mass. Axoneme number 1 is apparently complete, but has three primary derivatives attached to its periaxonemal sheath. Other axonemes (2 and 3 ) show increasing degrees of disruption. Isolated fragments of axonemal elements can be noted at the unnumbered arrows. A Golgi body $(G)$ is depicted at the right. $\times 75,000$.

Figure 6. Section through an aggregation of early spermatid mitchondria representing the closest approach to nebenkern formation seen in mutant ms (1)6S. This configuration is comparable to a stage midway between Stages 1 and 2 of normal spermatid differentiation. $x$ 24,000.

Figure 7. A section through a spermatid bundle in which the nebenkern derivatives show paracrystalline body (PB) formation comparable to stage 8 of normal differentiation. Note groups of microtubules (arrows) cut in transverse section. $\times 46,000$.

Figure 8. A longitudinal section through a mutant spermatid showing an elongate nebenkern derivative (ND) and some of the numerous microtubules (MT) that apparently run the full length of the cytoplasmic masses. $\times 46,000$.

Figure 9. A section through a mutant testis following treatment with Colcemid. Nuclei (N) are irregular as is chromatin condensation. Mitochondria (M) are in loop-shaped profiles. Microtubules are lacking and elongation of the cytoplasm does not occur. $x 9,400$. 


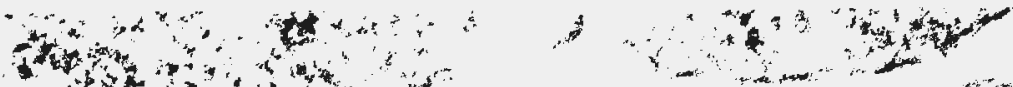

(1)

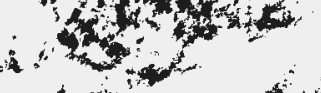

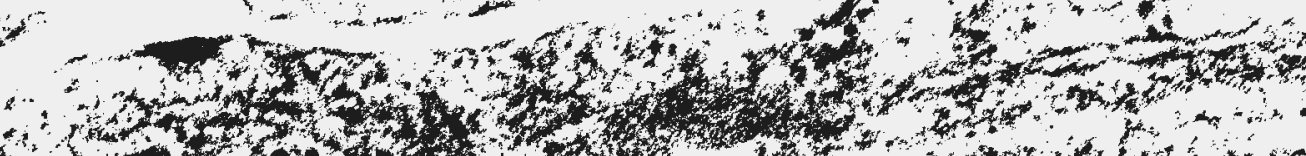

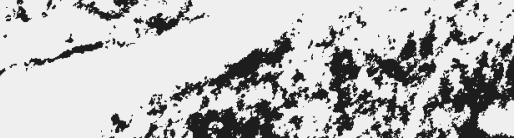
$x^{2}+2$

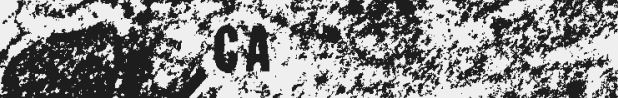

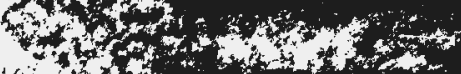

4,27

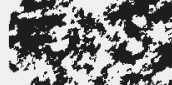

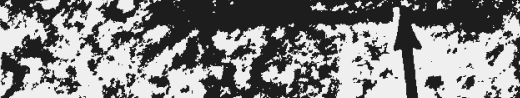

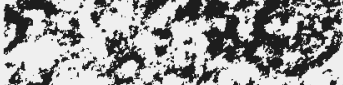

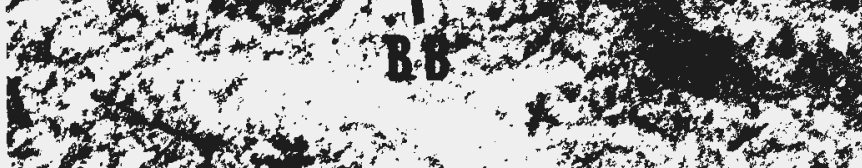

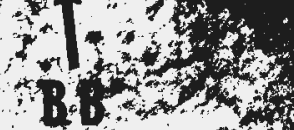

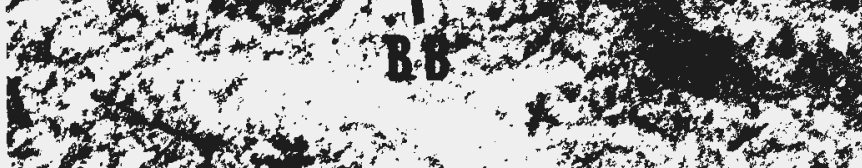

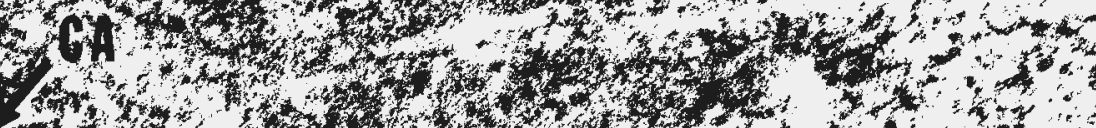
4

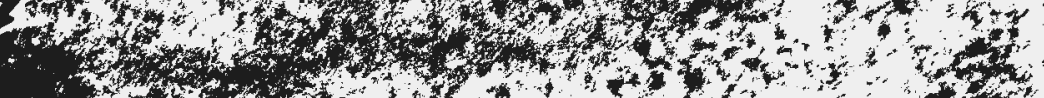
1.2.

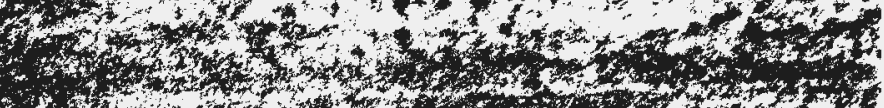

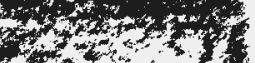

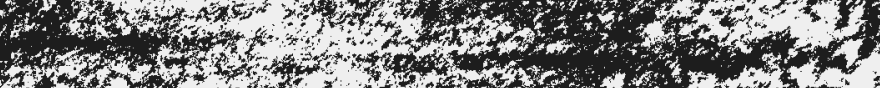

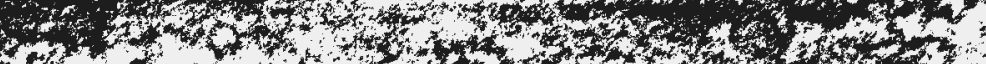

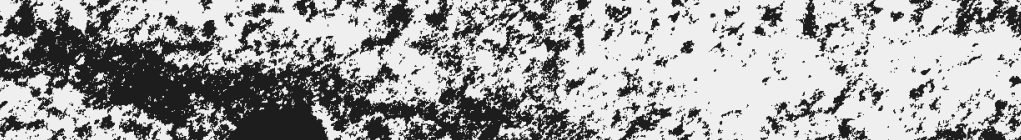

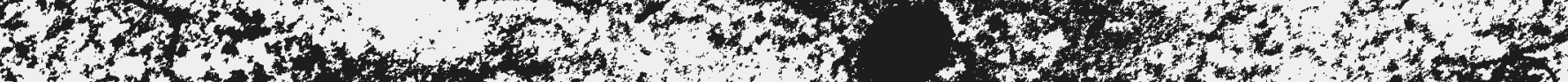

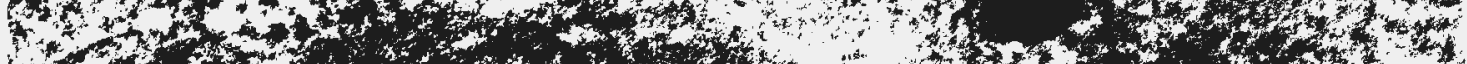

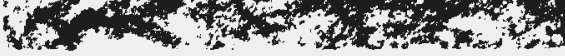
$2 x^{2}$

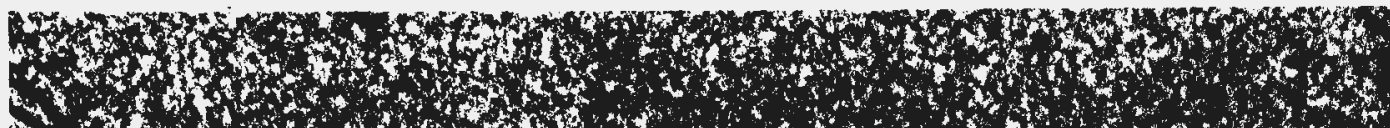

(1)

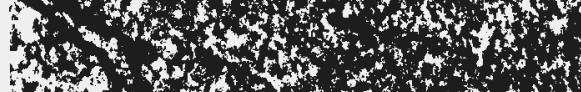

s.t.

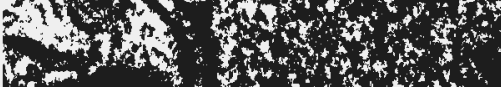

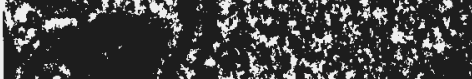

6

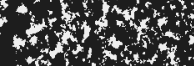

indy

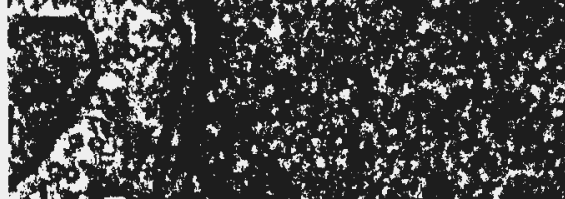

$2+x^{2}$

1

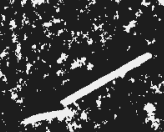

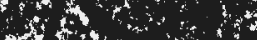

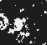

$6+2 x$

ats

$+x+4$

a) +28

000 of

$+40+20$

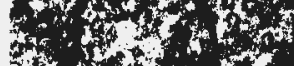

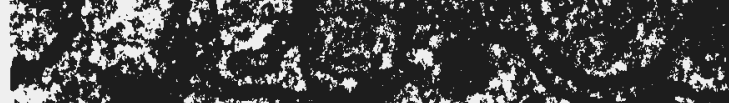

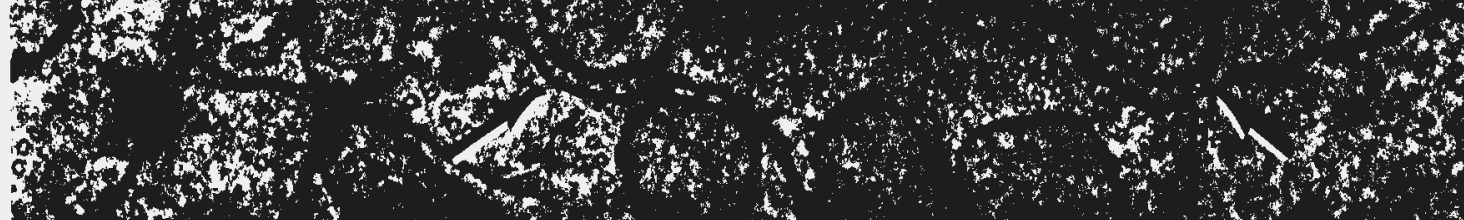

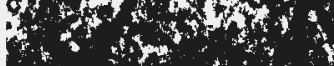

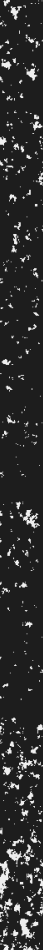

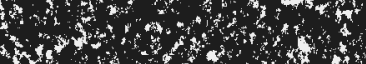




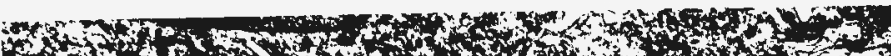

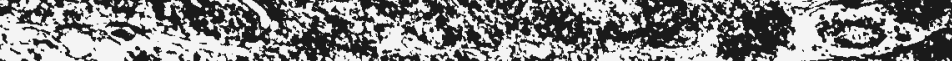

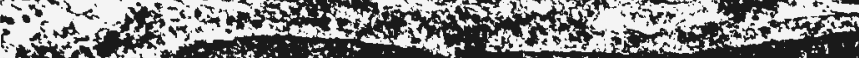

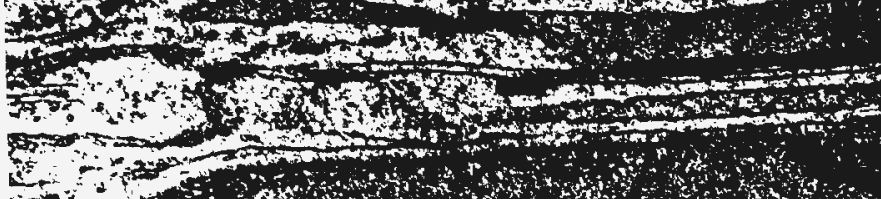

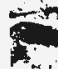

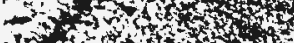

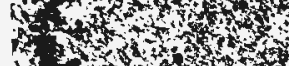

10
3

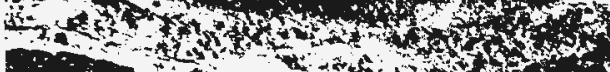

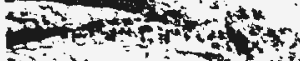

atsond

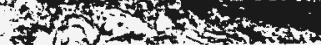

$=x+1$

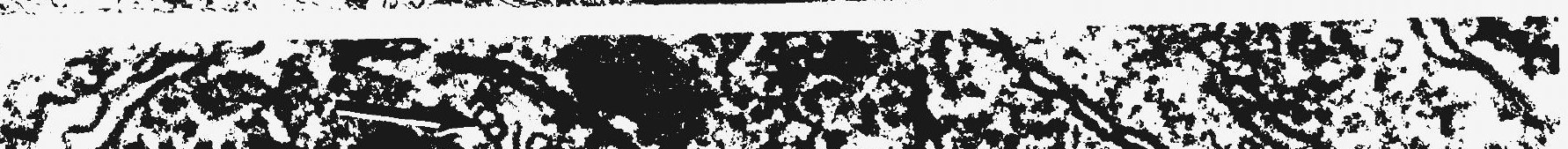

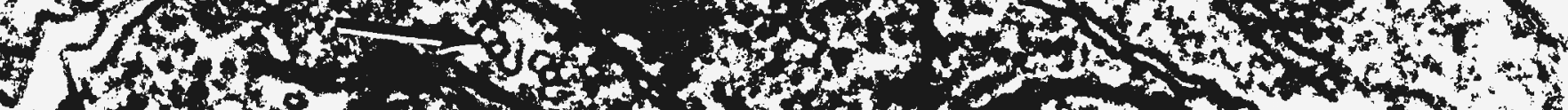

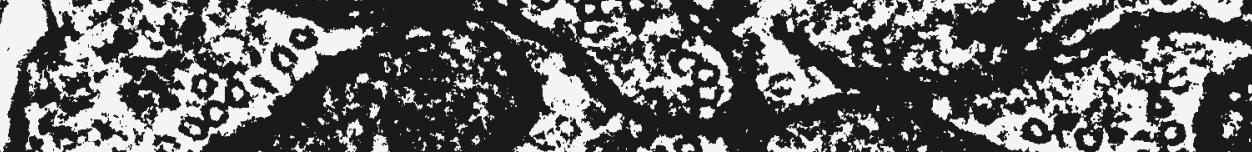

forsto

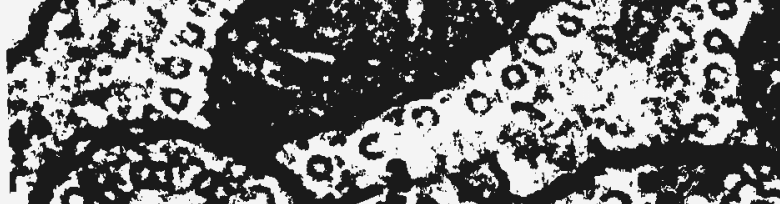

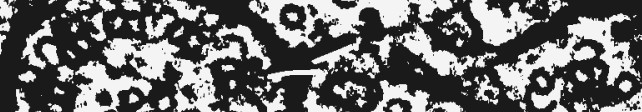

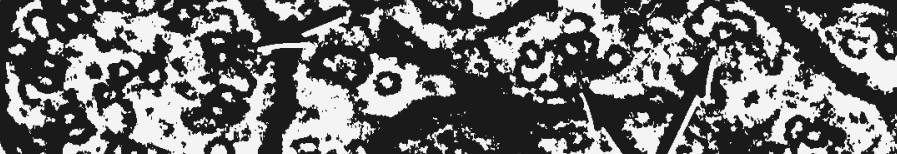

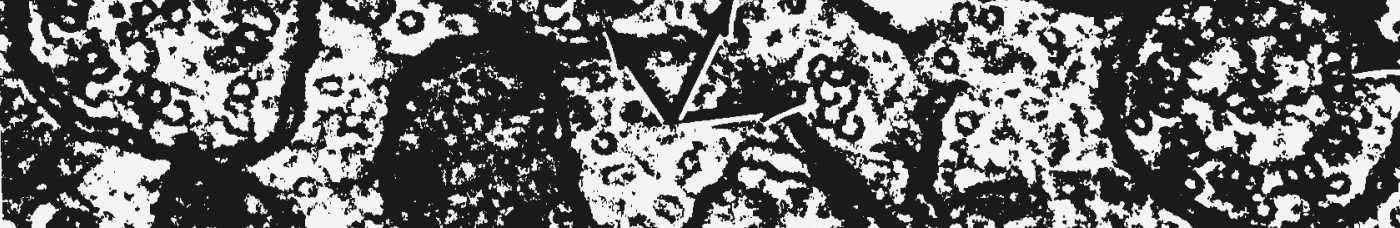

$x_{x \rightarrow 1}$

$+4048$

o.

$4 x+2+3$

on $\quad y+2$

d) +3

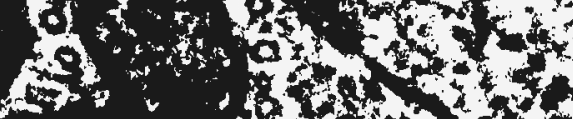

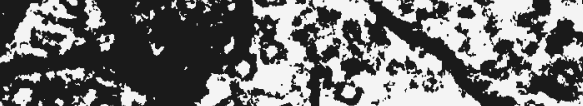

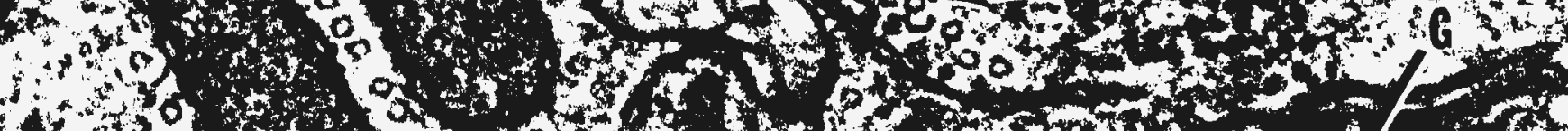

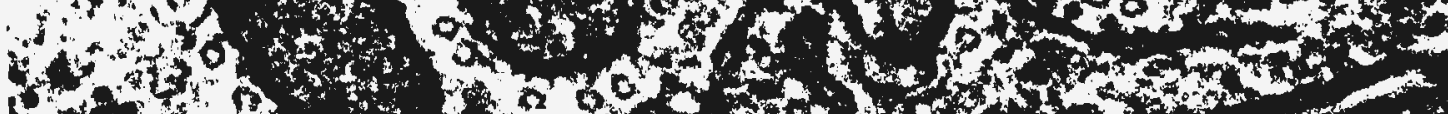

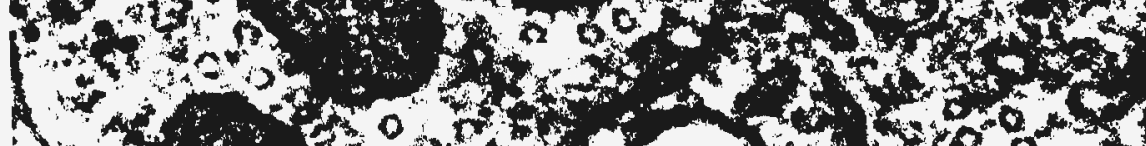

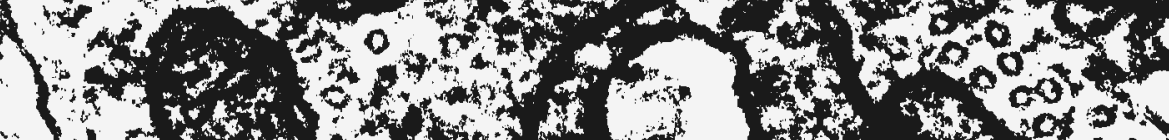

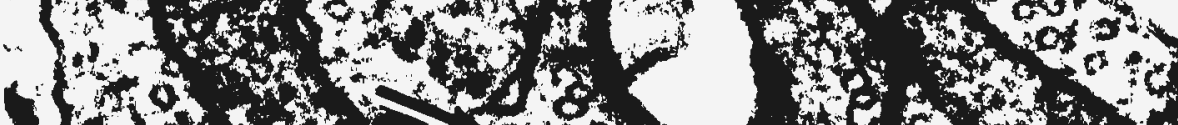
(4)

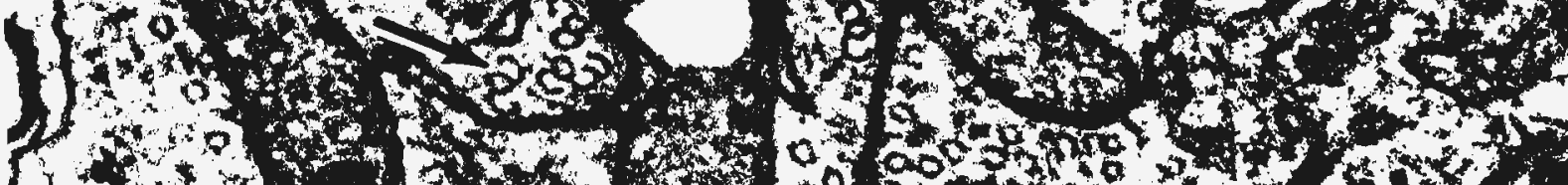

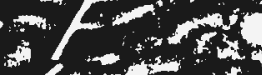

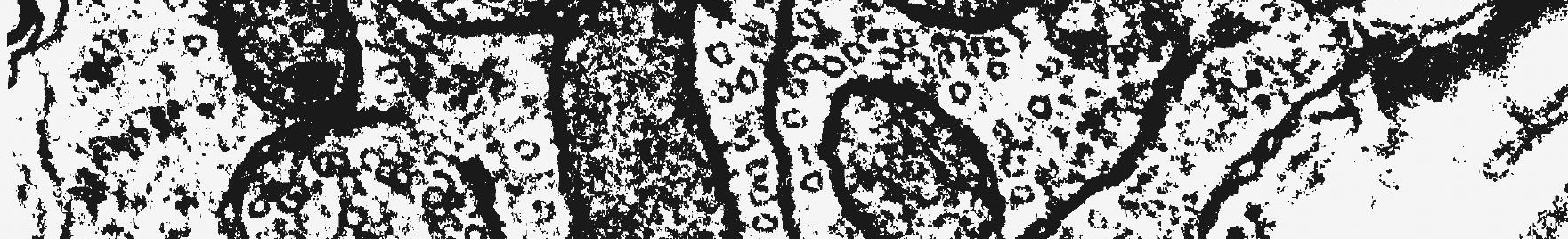

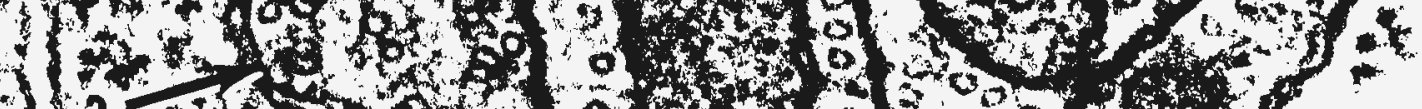

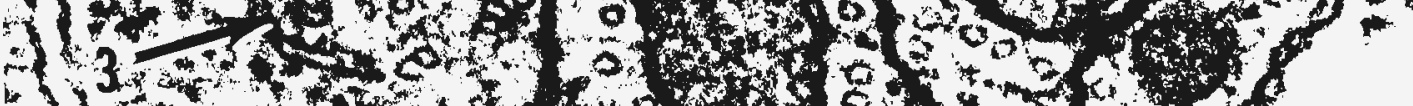

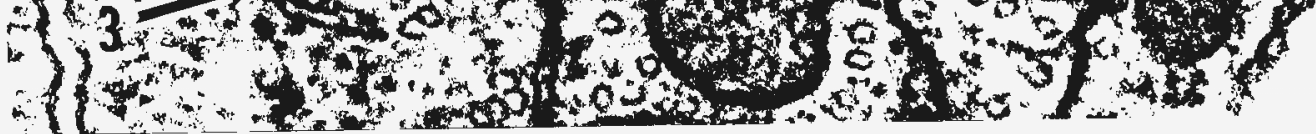




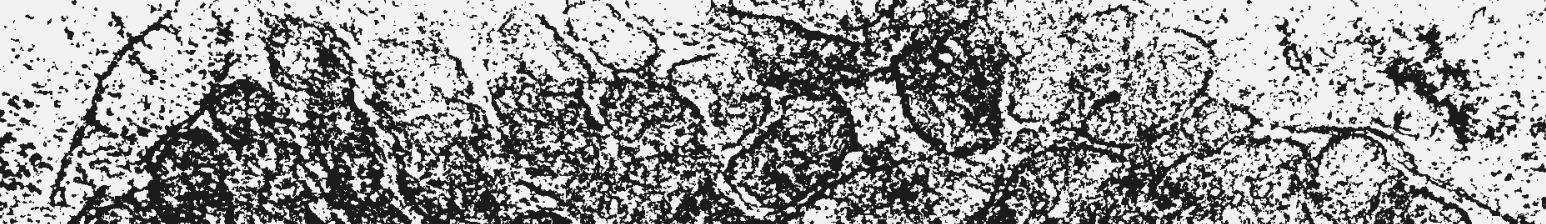

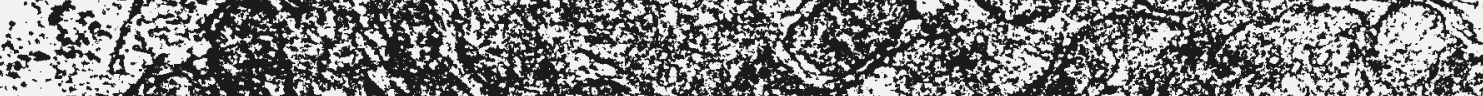

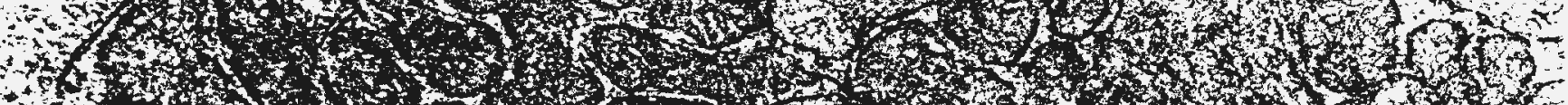

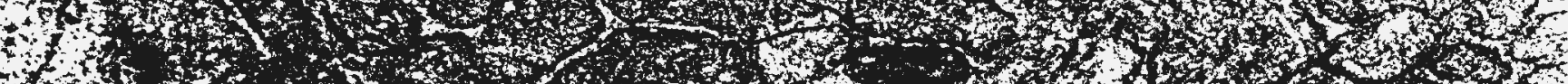

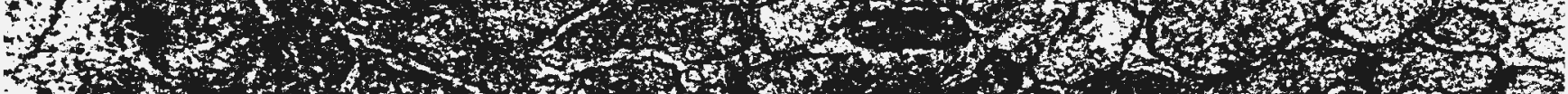

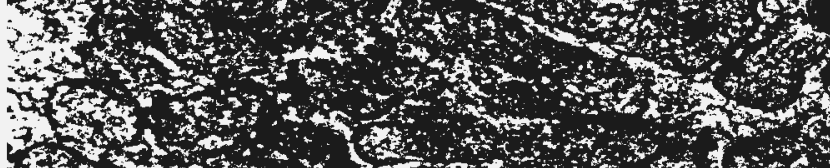

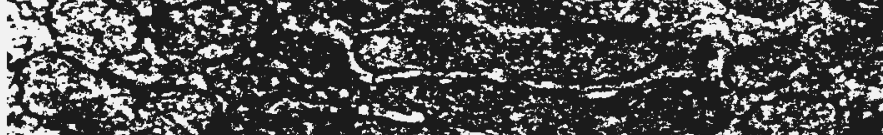

\section{tis}

3

sito

on

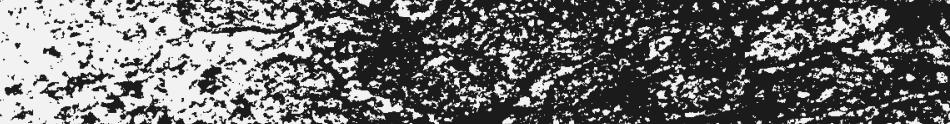

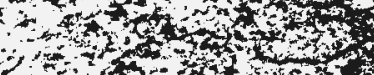

tom,

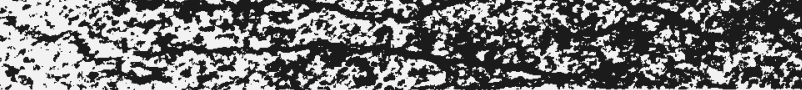

$+\frac{1}{2}$

betet ?

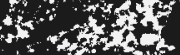
3.1.6. (1)

W

3.

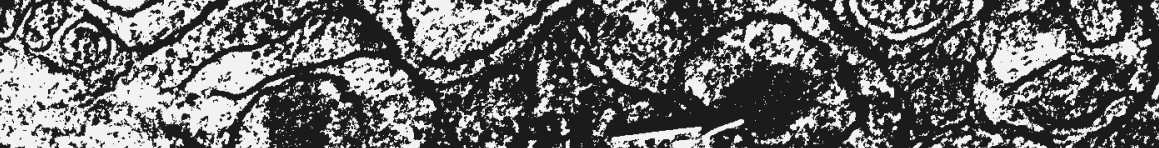

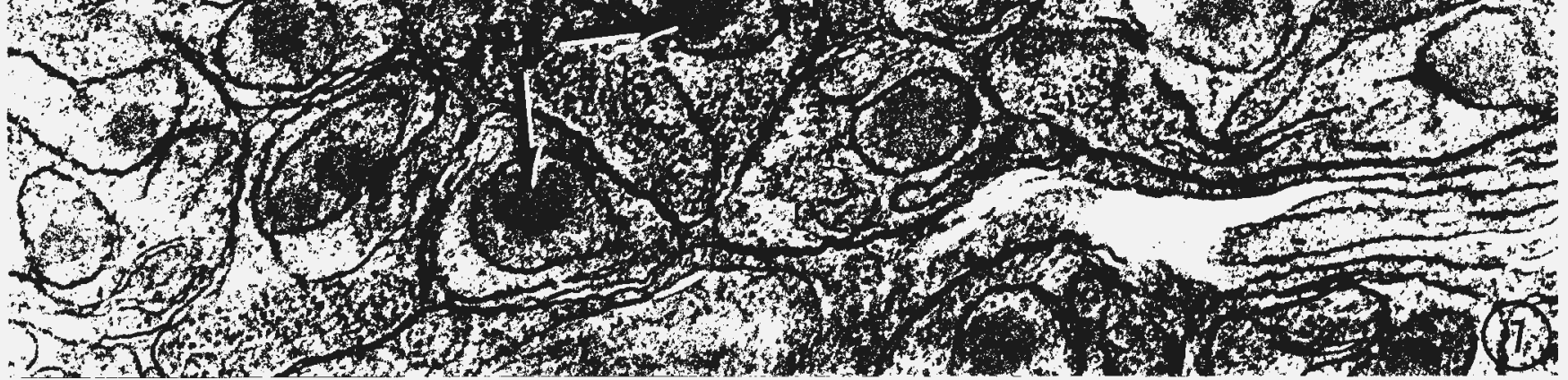




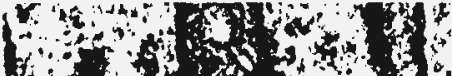

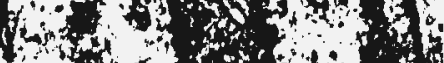

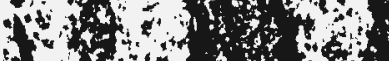

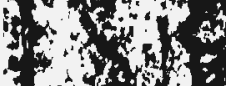

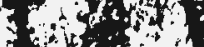

30

t $12 x+1$

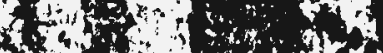

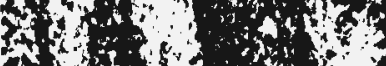

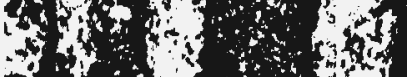

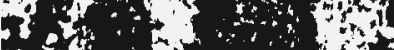

$\because+20$ the

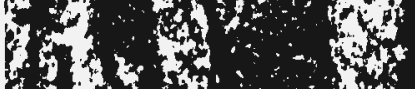

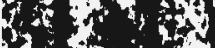

fis in:

15:4 o 30

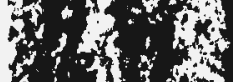

2. $3 x+3$

(to

at.

$+60$

Fto

aty

xes

(4)

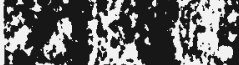

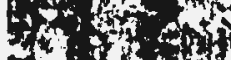

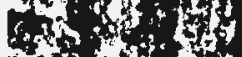

$+3$

4

sis

X.

,

$\therefore t$

Now $x$

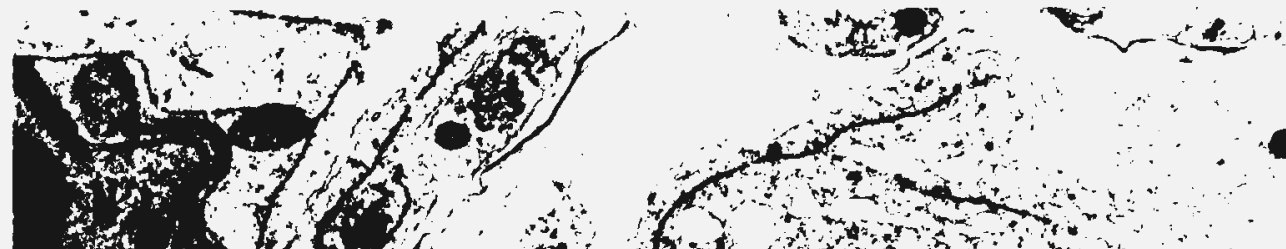

1. 35

at +4

ation

$+4$

$(6)$

$-\lim _{x \rightarrow \infty}$

of $f x^{2}$
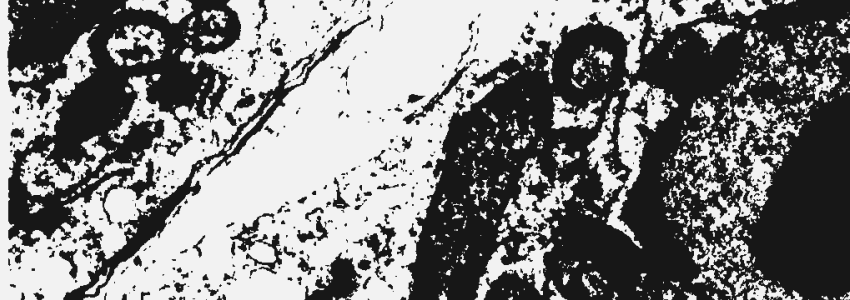

(1)

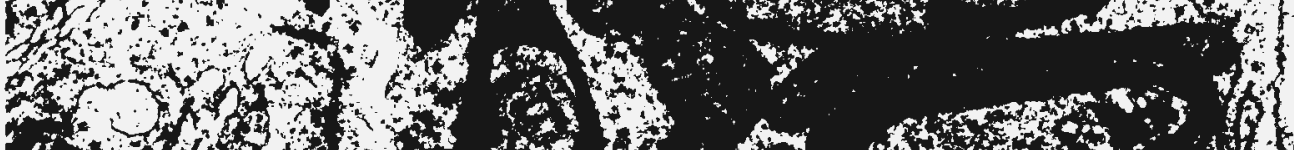
w of

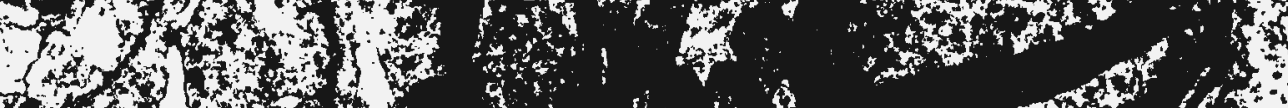

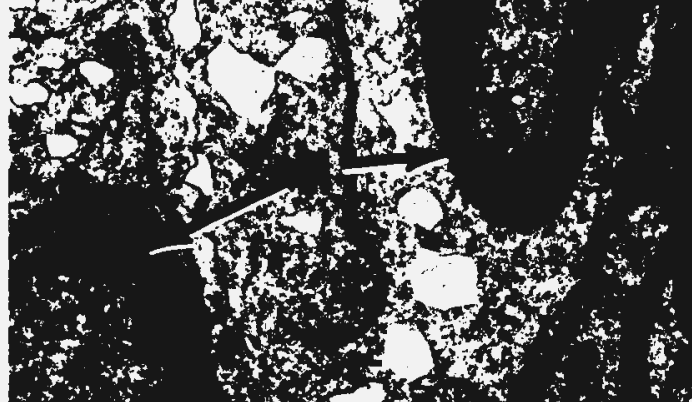

(1) $-1,4$

3i. 10 th

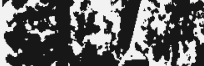

Het

in

a. 1,1

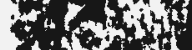

$+10+1$

t.

$\frac{4}{4 x y}$
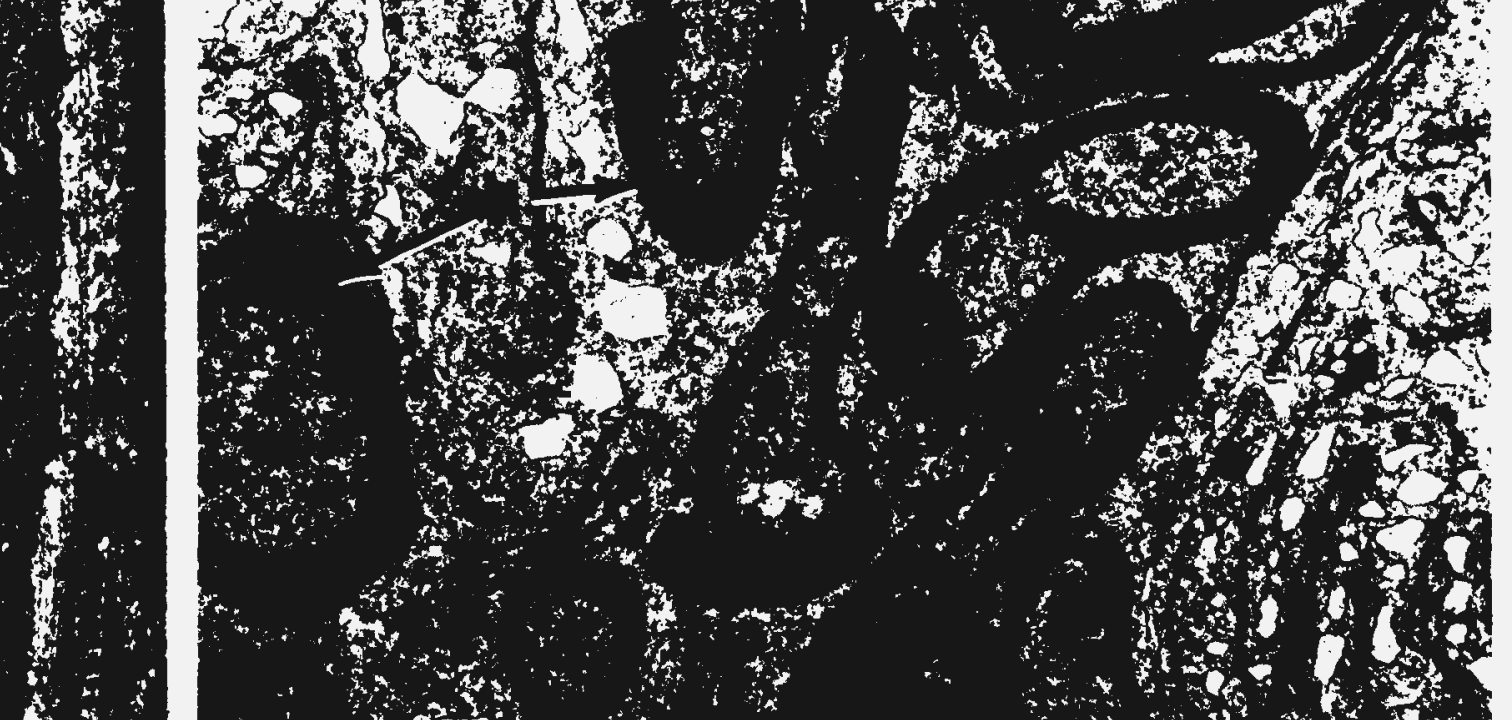

istes

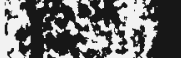

ing

ats

$x y$

sintis

Pand

Fo

$-2+n$

tot

3

situty
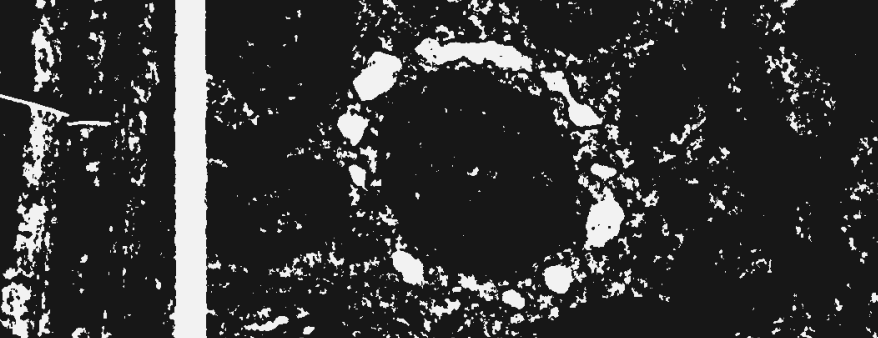

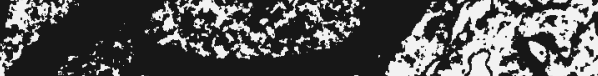

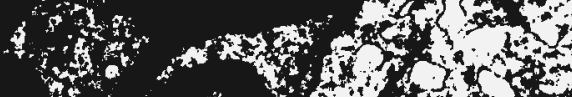
$y^{5}$

a. $30 \mathrm{cot}$

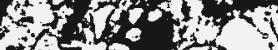

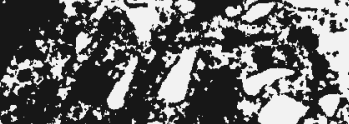

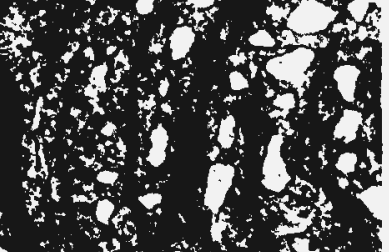
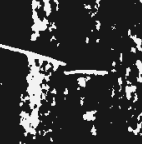

tis

8

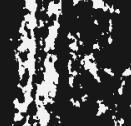

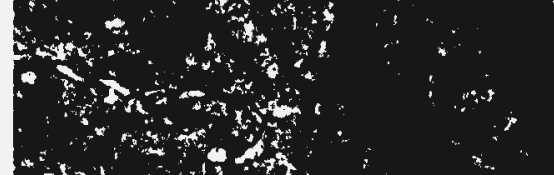

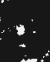

ros

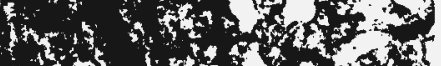

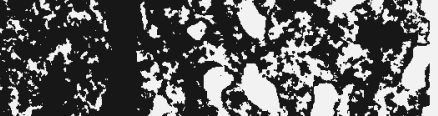

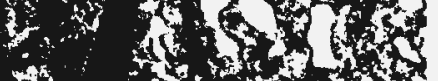

$4 x, 2+2+2$

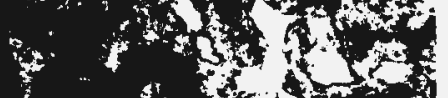

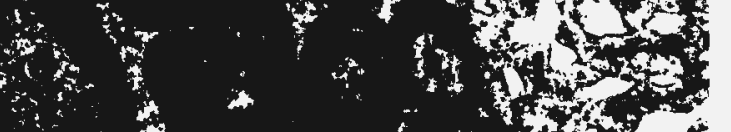

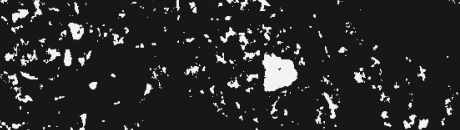

Het a n

to k

(3)

He

$8(1,2)$

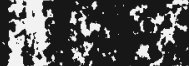

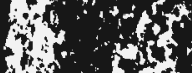

in,$x$

,

a

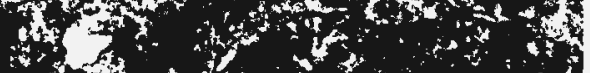

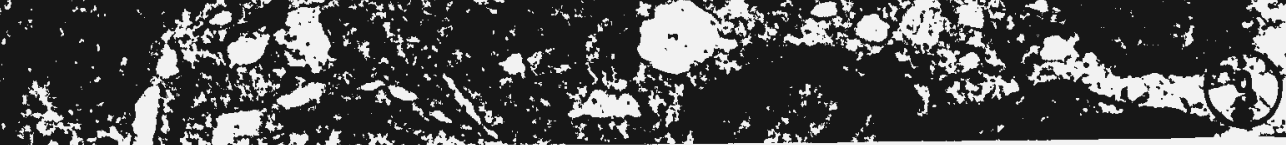

